\title{
Paul Lorenzens Weg von der Mathematik zur Philosophie - Persönliche Erinnerungen
}

\author{
Kuno Lorenz
}

Es gibt mittlerweile schon zahlreiche, auf gründlichen Recherchen und großenteils auch auf persönlichen Begegnungen beruhende Arbeiten zu den Stadien im Lebensweg Paul Lorenzens. ${ }^{1}$ Ergänzend dazu möchte ich im Folgenden einige unsystematische persönliche Erinnerungen vortragen, die vielleicht noch mehr Licht auf den in seiner Person verkörperten besonderen Zusammenhang von Mathematik und Philosophie werfen.

Ich hatte mein Studium in den Fächern Mathematik und Physik in Tübingen begonnen (SS 1951 - WS 1952/53) und zum Ende des Wintersemesters 1953/54 an der Universität Hamburg den ersten Studienabschnitt mit dem Vordiplom in beiden Fächern (theoretische Mathematik bei Helmut Hasse und praktische Mathematik bei Lothar Collatz) abgeschlossen. Nach dieser von einer Studienunterbrechung begleiteten Zäsur schrieb ich mich mit denselben Fächern zum WS 1954/55 an der Universität Bonn ein. Was war der Grund? Ein Ferienkurs im Oktober 1954 in Villigst bei Schwerte/Ruhr (heute Ortsteil von Schwerte), dem Sitz des Evangelischen Studienwerks.

Ich hatte mein Abitur 1950 in der DDR abgelegt und verdankte >Villigst die Förderung meines Studiums in der BRD durch ein Stipendium und andere Angebote, zu denen auch Ferienkurse gehörten. Der Kurs, um den es hier ging, war von einem schon fortgeschritteneren Villigster Studenten der Mathematik vorbereitet worden, und ich wurde darauf im Vorfeld aufmerksam. Das Thema: die von Paul Lorenzen an der Universität Bonn in dieser Zeit neu entwickelte operative Logik im Zusammenhang einer grundsätzlich auf den Strichkalkül des Zählens gegründeten operativen Arithmetik.

\footnotetext{
Kuno Lorenz

Universität des Saarlandes, Saarbrücken, Deutschland, E-mail: klorenz@mx . uni-saarland .de

${ }^{1}$ Vgl. stellvertretend für viele weitere: C. Thiel, „Paul Lorenzen (1915-1994)“, Zeitschrift für allgemeine Wissenschaftstheorie 27 (1996), 1-13; sowie ders., „Paul Lorenzen“, in: Enzyklopëdie Philosophie und Wissenschaftstheorie (ed. J. Mittelstraß), Band 5, Stuttgart/Weimar: J. B. Metzler, ${ }^{2}$ 2013, 112-115.
} 
Lorenzen hatte zugesagt, diesen Kurs selbst zu leiten. Ich meldete mich an und begegnete ihm dort zum ersten Mal.

Im Vergleich mit der Art, wie ich zuvor mit einigen mathematischen Gebieten in Vorlesungen und Übungen vertraut gemacht worden war, wurde mir schon damals in Villigst deutlich, daß Lorenzen uns, die Teilnehmer des Ferienkurses, mit einem für uns neuen Denken über mathematische Gegenstände zu konfrontieren suchte. Nicht um Versuche, etwas schon Bereitliegendes zu entdecken, das es anschließend durch Beweise in seiner Existenz unter einer Beschreibung zu sichern gelte, sollte es gehen. Vielmehr handelte es sich darum, diese mathematischen Gegenstände allererst zu konstruieren, um dann daraus die für sie eigentümlichen Eigenschaften zu ermitteln. Aber natürlich standen keine handwerklichen Konstruktionen zur Debatte, sondern gedankliche, allein durch symbolische Zeichen vermittelte; es ging nicht um reale Konstruktionen, sondern um ideale. Viel später erst begriff ich, daß mit Lorenzen verfahrensbezogen ein moderner Platon hinter dem Vorlesungspult stand und kein moderner Aristoteles.

Die Entscheidung, an der Universität Bonn das Studium fortzusetzen, um insbesondere bei Lorenzen zu lernen, war gefallen. Damals wußte ich manches noch nicht, z.B. daß Lorenzen 1939 - nach der im Jahr zuvor abgelegten Promotion bei Helmut Hasse in Göttingen - als Wissenschaftlicher Assistent bei Wolfgang Krull in Bonn seine Hochschullaufbahn begonnen hatte. Oder daß nach der kriegsbedingt verspäteten Habilitation für Mathematik 1946 in Bonn seine im Jahr 1952 erfolgte Ernennung zum außerplanmäßigen Professor die Kennzeichnung ,für Mathematik und Geschichte der Mathematik' trug. Ich wußte auch nicht, daß die Habilitationsschrift ,Über halbgeordnete Gruppen' ihm schon 1948/49, nur dreieinhalb Jahre nach Kriegsende, eine Gastdozentur an der Universität in Cambridge eingetragen hatte. Diese Einladung war auch eine Antwort auf die große internationale Anerkennung gewesen, die sich Lorenzen mit dem 1951 vorgelegten Beweis der Widerspruchsfreiheit der verzweigten Typenlogik in den Principia Mathematica von Alfred North Whitehead und Bertrand Russell erworben hatte. Dieser Beweis beruhte auf der Verwendung der in der Habilitationsschrift erstmals eingesetzten Halbformalismen als entscheidendem Beweismittel. Mit dem derart öffentlich dokumentierten Schritt heraus aus der bloß innerfachlichen mathematischen Arbeit hin zu einer die Grundlagen der Mathematik sowohl systematisch als auch historisch ausdrücklich thematisierenden Forschung wurde der erste Schritt Lorenzens auf dem Wege von der Mathematik zur Philosophie sichtbar. Wichtig sind in diesem Zusammenhang die bedeutenden Arbeiten zur antiken griechischen Mathematik des ebenfalls an der Universität Bonn tätigen Phänomenologen Oskar Becker. Ihnen galt Lorenzens andauerndes, von intensivem Gedankenaustausch begleitetes wissenschaftliches Interesse.

Diese Zusammenhänge waren dem Studenten der Mathematik im zweiten Studienabschnitt, der ich zu Beginn des WS 1954/55 gewesen war, noch verborgen geblieben. Sie kamen auch nicht zur Sprache, als unter der Leitung 
von Lorenzen in einem Hauptseminar, an dem ich teilnahm, die zentralen Ideen der Principia Mathematica erarbeitet werden sollten. Allein das Projekt eines operativen Aufbaus von Arithmetik und Logik faszinierte mich. Es war in der damals gerade im Druck befindlichen Monographie Einführung in die operative Logik und Mathematik von Lorenzen vorgestellt und ein erhebliches Stück weit ausgeführt worden. Diese Faszination galt auch der in einer Vorlesung angebotenen Version einer Einführung in die Differentialgeometrie mit den Mitteln von Tullio Levi-Civitas Tensordarstellung, von der ich eine von Lorenzen durchgesehene und damit approbierte Vorlesungsnachschrift erstellt habe. Doch erst mit der für die Zulassung zum Staatsexamen im WS 1956/57 bei Krull angefertigten Arbeit „Über Strukturverbände von Verbandsgruppen" begab ich mich auf ein einst von Lorenzen betretenes Gebiet, um bei der Einarbeitung in dieses Gebiet zu erfahren, daß es Lorenzen gewesen war, der dort bereits wichtige Wege gebahnt hatte.

Aber noch etwas anderes ereignete sich gleich zu Beginn des WS 1954/55 in Bonn, dessen Bedeutung erst im Laufe der Jahre sichtbar wurde: die Begegnung von Lorenzen mit dem um fast genau zehn Jahre älteren philosophischen Kollegen Wilhelm Kamlah. Kamlah hielt einen auch von mir besuchten Gastvortrag über das Thema „Was ist Wahrheit?“ Zwar gehörte ich nicht zu den Zeugen des vermutlich ersten philosophischen Gesprächs zwischen den beiden Männern - ich weiß nicht einmal, ob es solche Zeugen überhaupt gegeben hat -, wohl aber läßt die nachfolgende Entwicklung einer Übereinstimmung des Interesses an einer grundsätzlichen, von Komplementarität geprägten Gemeinsamkeit bei der Neubestimmung philosophischer Arbeit diese erste Begegnung als folgenreich für den Weg Lorenzens von der Mathematik zur Philosophie erscheinen.

Es ist die Grundverschiedenheit des wissenschaftlichen Hintergrundes bei Kamlah und Lorenzen, der Theologie mit ihrem Methodenschwerpunkt (linguistischer) Hermeneutik bei Kamlah und der Mathematik mit ihrem Methodenschwerpunkt (logischer) Grammatik bei Lorenzen, die zu einer besonderen Herausforderung für die künftige philosophische Arbeit beider Gelehrter werden wird.

Zunächst jedoch erging an Lorenzen der Ruf auf einen Lehrstuhl für Philosophie an der Universität Kiel (1956), den er annahm. Diese institutionelle Wende zur Philosophie wurde für ihn, nach seiner von Kamlah betriebenen Berufung nach Erlangen (1962), zum Sprungbrett für den Aufbau des unter der Bezeichnung „Erlanger Schule“ bekannt gewordenen Zentrums für konstruktive Philosophie und Wissenschaftstheorie. Damit wiederholt sich auf eigentümliche Weise im Abstand etwa einer Generation die Rolle eines philosophischen Lehrstuhls der Universität Kiel als Sprungbrett für den Aufbau einer besonderen logisch-philosophischen Schule an einer anderen Universität Deutschlands. Ich denke dabei an Heinrich Scholz (1884-1956), der von einem theologischen Lehrstuhl für Religionsphilosophie und systematische Theologie an der Universität Breslau auf einen philosophischen Lehrstuhl an der Universität Kiel berufen worden war (1921), um schließlich 
binnen weniger Jahre im Zuge eines Rufs an die Universität Münster in Westfalen dort das erste deutsche Zentrum für Mathematische Logik und Grundlagenforschung aufzubauen (1928). Der Rolle von Platon als Vorbild für die paradigmatisch zu verstehende wissenschaftstheoretische Arbeit von Lorenzen im Rahmen der Erlanger Schule korrespondiert die Rolle von Aristoteles als Vorbild für die kumulativ zu verstehende formallogische Arbeit von Scholz im Rahmen des Zentrums für logische Grundlagenforschung.

Schon im ersten Kieler Jahr erreichte Lorenzen die ehrenvolle Einladung, für ein Studienjahr (1957/58) als Visiting Member nach Princeton (New Jersey) an das Institute for Advanced Study zu kommen. Ich wiederum hatte 1957 mein Staatsexamen in den Fächern Mathematik und Physik an der Universität Bonn abgelegt und mich zugleich erfolgreich um ein DAAD-Stipendium für das Studienjahr 1957/58 an der Universität Princeton beworben, wobei ich die Zulassung als Visiting Fellow am Graduate College für das Department of Mathematics ebendort bereits vorher bekommen hatte. Mein Ziel war, neben meinem Interesse an gründlicher Weiterbildung auf dem Feld der abstrakten Algebra, insbesondere bei Emil Artin, vor allem einem intensiven Studium der mathematischen Logik bei Alonzo Church nachzugehen. Ich konnte also zur selben Zeit in Princeton arbeiten wie Lorenzen und hatte dank eines Fulbright Travel Grant sogar das Glück, auf demselben Schiff wie er im Frühherbst 1957 nach den USA überzusetzen.

Während einiger Gespräche, die ich mit Lorenzen über Probleme konstruktiver Beweisführungen dort, wo sonst der Wohlordnungssatz oder das mit ihm grundsätzlich gleichwertige Zornsche Lemma in Anspruch genommen wird, führen konnte, festigte sich mein Entschluß, nach dem Ende des Studienjahres in Princeton zu ihm nach Kiel zu gehen, um an einer Dissertation über Konstruktivitätsprobleme in der Idealtheorie zu arbeiten. Lorenzen hatte im übrigen schon angefangen, in Zusammenarbeit mit John Myhill einen Aufsatz zu verfassen, mit dem sich das mögliche Zusammenspiel von axiomatischer und konstruktiver Methode sinnfällig demonstrieren ließ. ${ }^{2}$ Der möglichen Alternative, zu versuchen, unter der Supervision von Artin an einer Dissertation im Anschluß an meine Staatsexamensarbeit über Verbandsgruppen zu arbeiten, ließ sich damals nicht weiter nachgehen, weil Artin gerade einen Ruf von Princeton zurück an die Universität Hamburg erhalten hatte, dem er dann auch gefolgt war. Dieser Ruf gehört zu den selten gebliebenen Akten versuchter 'Wiedergutmachung für die gleich nach dem Beginn der Naziherrschaft in Deutschland beginnende Entrechtung der Juden, zunächst >bloß< durch Entfernung aus dem Staatsdienst, wie im Falle Artins.

In einem der genannten Gespräche mit Lorenzen berichtete er auch von einer während eines internationalen Kolloquiums zur axiomatischen Methode am Jahresende 1957/58 in Berkeley 3 geführten intensiven Ausein-

${ }^{2}$ P. Lorenzen \& J. Myhill, „Constructive Definition of Certain Analytic Sets of Numbers", Journal of Symbolic Logic 24 (1959), 37-49.

3 Vgl. L. Henkin/P. Suppes/A. Tarski (eds.), The Axiomatic Method with Special Reference 
andersetzung mit Alfred Tarski über den Begriff der Definitheit prädikativer Ausdrücke, der in Lorenzens damals vieldiskutierter Einführung in die operative Logik und Mathematik eine Schlüsselrolle spielt. Lorenzen ließ sich davon überzeugen, daß dieser der Absicht nach im Vergleich zum Begriff der Entscheidbarkeit logisch schwächere Begriff der Definitheit seinerseits nicht in dem Sinne einwandfrei definiert ist, daß eine Kontrollmöglichkeit existierte, ob eine behauptete Definitheit einer Begriffsbildung tatsächlich vorliegt oder nicht. Genau diese Lücke weist auch der allein auf dem Nachweis der Eliminierbarkeit von Regelanwendungen fußende Grundbegriff der Zulässigkeit von Kalkülregeln auf, wenn, wie in der operativen Logik notwendig, den strengen Begriff eines Kalküls sprengende Metakalküle, noch dazu beliebiger Stufe, in Betracht gezogen werden müssen. Irgendwelche sinnvollen Vollständigkeitsbeweise lassen sich deshalb für die operative Logik nicht führen.

Damit wurde Lorenzen für seinen nächsten Schritt auf dem Weg von der Mathematik zur Philosophie bestärkt: einer Verankerung mathematischer Begriffsbildungen in der anthropologischen Fähigkeit zur Vernunft durch von ihr geleitete Auseinandersetzungen, also Dialoge. Definitheit soll durch Dialogdefinitheit präzisiert werden. Aussagen, besonders sinnfällig in der Mathematik, werden nicht mehr für durch Wahr- oder Falschsein charakterisiert gehalten, und auch nicht durch Beweis- oder Widerlegbarkeit; vielmehr sollen sie dadurch charakterisiert sein, daß sich um sie eine strukturell präzisierte Auseinandersetzung, d.h. ein Dialog, führen läßt. All das wurde mir erst viel später bewußt, während meiner eigenen, unter seiner Betreuung stehenden Arbeit an einem spieltheoretischen Zugang zur formalen Logik. Schon im September 1958 wird Lorenzen in seinem Beitrag „Logik und Agon" auf einem Philosophie-Kongreß in Venedig diesen Schritt öffentlich machen. 4

Ebenfalls 1958, im Frühjahr, noch vor der Rückkehr nach Kiel, waren Lorenzen - und ich mit ihm - zu einer Arbeitstagung bei Haskell B. Curry am Department of Mathematics der Pennsylvania State University in State College eingeladen, während der es unter anderem um die Differenz zwischen einem axiomatischen und einem konstruktiven Aufbau mathematischer Theorien, bei Curry einer >théorie des obs«, ging. 5 So ließ sich noch einmal deutlich machen, welch wichtige Rolle der Unterschied zwischen einem ontologischen und einem pragmatistischen Verständnis mathematischer Theoriebildung spielt, wenn es den vermeintlichen Gegensatz von axiomatischem und konstruktivem Vorgehen aufzulösen gilt. Das blieb auch der Hintergrund für die zentrale Fragestellung bei der Behandlung des

to Geometry and Physics: Proceedings of an International Symposium Held at the University of California, Berkeley, Dec. 26, 1957 - Jan. 4, 1958, Amsterdam 1959.

4 In den Atti del xII Congresso Internazionale di Filosofia (Venezia, 12-18 Settembre 1958) IV (Logica, linguaggio e communicazione), Florenz 1960, 187-194.

5 Vgl. H. B. Curry, Leçons de logique algébrique, Paris 1952 - „obs“ steht für „unspecified objects". 
1960 erschienenen Buches Word and Object von Willard Van Orman Quine in einem Oberseminar in Kiel. Lorenzen hatte bereits in einem Gespräch mit Quine in Harvard noch im Jahr 1958 von der bevorstehenden Publikation erfahren und sich für das geplante Oberseminar in Kiel entsprechend gut vorbereiten können. In ihm sollte geklärt werden, welchen Sinn es haben kann, sich beim Aufbau einer Wissenschaftssprache auf die traditionelle Alternative einzulassen, es sei entweder >die Welt $<$ - man ergänze: des Geistes oder der Natur - oder der sie erforschende Mensch, die das Vorgehen bestimmten.

Schon bald nach den im WS 1958/59 begonnenen Vorbereitungen zu meiner geplanten Dissertation stellte sich heraus, daß die logischen Grundlagen zur Behandlung von Konstruktivitätsproblemen in der Mathematik gerade dort, wo keine Beschränkung auf allgemeinrekursive Mathematik beabsichtigt ist, noch nicht standsicher sind. Das lag vor allem daran, daß der zunächst noch weitgehend anschaulich durch Angreifbarkeit seitens eines Opponenten und Verteidigbarkeit seitens eines Proponenten erläuterte Begriff der Dialogdefinitheit einer Aussage noch einer genauen begrifflichen Bestimmung entbehrte. Die Idee eines nach präzisen Regeln ablaufenden Dialogspiels um eine Aussage, einer Folge von Angriffen gegen sie und Verteidigungen von Aussagen auf solche Angriffe, als Grundlage für eine Ermittlung ihrer Geltung (definiert als Existenz einer Gewinnstrategie für den Proponenten) bedurfte noch einer zuverlässigen Realisierung. Dabei standen zunächst ausschließlich logisch zusammengesetzte und nicht logisch einfache Aussagen, und damit die Rolle der Logik innerhalb der Mathematik, im Fokus. Ich ließ daher nach der Rückkehr von einer mit Lorenzen und seinem mathematischen Kollegen Friedrich Bachmann gemeinsam unternommenen Fahrt nach Budapest zum 2. Ungarischen MathematikerKongreß (Második Magyar Matematikai Kongresszus, 24.-31. August 1960) meinen ursprünglichen Dissertationsplan fallen und entschloß mich, im Einverständnis mit Lorenzen, nach einem spieltheoretischen Fundament von Logikkalkülen und damit zugleich von der als methodisch für nicht hinreichend fundiert erkannten operativen Logik zu suchen. Darüber hinaus stellte sich heraus, daß so auch die Fessel einer Anwendbarkeit auf nur aus Ableitbarkeitsaussagen logisch zusammengesetzte Aussagen, wie sie bei der operativen Logik der Fall ist, gesprengt werden konnte. Es ging um den Begriff der Wahrheit in voller Allgemeinheit und damit um den Begriff einer Aussage im allgemeinen, natürlich unter Einschluß des Wahrheitsbegriffs in den Wissenschaften; es waren nicht nur die Mathematik, und über sie hinaus die Logik als bloßes Werkzeug für diese, betroffen.

Damit war Lorenzen den ganzen Weg von der Mathematik zur Philosophie gegangen, auch wenn diese zunächst nur in Gestalt konstruktiver Wissenschaftstheorie unter Einschluß der Geschichte wissenschaftlichen Denkens konzipiert schien und die normativen Aspekte der Wissenschaften ebenso wie die der Ethik und der Politik erst Jahre später ebenfalls ins 
Zentrum von Lorenzens wissenschaftlicher Tätigkeit rückten. ${ }^{6}$ Gleichwohl gehörte zu den Zeichen für die Dringlichkeit kritischer Reflexion auf die Zusammengehörigkeit von wissenschaftlich fundierter Weltauffassung und vernünftig orientierter Lebensführung - so jedenfalls sehe ich es heute schon die damals, 1961, vom Promotionsausschuß an mich gestellte Frage, ob ich, als erster Promovend Lorenzens, einen Dr. phil. oder einen Dr. rer. nat. zu tragen wünsche. Das seit der Antike nie verlorengegangene Wissen um die Zugehörigkeit der Logik sowohl zur Philosophie als auch zu den Wissenschaften, und unter diesen besonders den exakten Wissenschaften, hatte eine zeitgemäße Gestalt gefunden.

Zum SS 1962 begann Lorenzens Lehr- und Forschungstätigkeit an der Universität Erlangen, geprägt von der Kooperation mit seinem Kollegen Kamlah - zu den sichtbaren Zeichen dafür gehörte das legendäre stets von beiden gemeinsam geplante und geleitete Oberseminar -, und ich konnte ihm als sein Wissenschaftlicher Assistent, von dem er stetige Arbeit an einer Habilitationsschrift erwartete, folgen.7 Dabei entwickelte sich schnell eine ganz ähnlich auf Komplementarität des akademischen Hintergrundes gegründete >dialogische`Zusammenarbeit von Jürgen Mittelstraß, damals Wissenschaftlicher Assistent von Kamlah, und mir. In den Folgejahren bis zu unserer beinahe gleichzeitig erfolgten Habilitation im WS 1968/69 führte sie nicht nur zu einer Reihe gemeinsam geschriebener Aufsätze, ${ }^{8}$ sondern dauert bis heute an, vor allem in Gestalt meiner Beteiligung an dem von Mittelstraß initiierten Langzeitprojekt einer Enzyklopädie Philosophie und Wissenschaftstheorie, die seit Ende 2018 in einer zweiten, gründlich überarbeiteten und erweiterten Auflage abgeschlossen vorliegt.

Bis zu meiner Berufung auf einen philosophischen Lehrstuhl an der Universität Hamburg 1970 gab es in Erlangen unter meiner Beteiligung eine Fülle von Unternehmungen Lorenzens mit dem Ziel, ein breiteres Verständnis für die Vorgehensweisen konstruktiver Logik und Wissenschaftstheorie als Formen wissenschaftlich sich äußernder Vernunft zu erreichen. Einige davon aus dem Jahr 1964 möchte ich herausgreifen. Sie lassen das immer weiter ausgreifende, von fachlichen Grenzen unbeeindruckte Interesse Lorenzens an einem dialogisch kontrollierten Denken besonders deutlich hervortreten.

\footnotetext{
${ }^{6}$ Dokumentiert u.a. durch den in Kiel entstandenen Band Die Entstehung der exakten Wissenschaften (Berlin/Göttingen/Heidelberg 1960); hingegen erst 1967/68 in den in Oxford gehaltenen John Locke Lectures - veröffentlicht unter dem Titel Normative Logic and Ethics (Mannheim/Zürich 1969) - die auch öffentlich sichtbar gewordene Konzentration auf Art und Rolle normativer Fragestellungen.

$7 \mathrm{Zu}$ den erstmals einer breiteren Öffentlichkeit zugänglich gemachten Ergebnissen gehört die von Lorenzen und Kamlah in mehrjährigen Diskussionen und Gesprächen mit Studierenden und Assistenten erarbeitete und von beiden gemeinsam verfaßte, mittlerweile als Gründungsschrift der `Erlanger Schule` geltende Logische Propädeutik oder Vorschule des vernünftigen Redens (Mannheim 1967).

${ }^{8}$ Einige dieser Aufsätze sind mittlerweile zugänglich in: K. Lorenz, Philosophische Variationen: Gesammelte Aufsätze unter Einschluß gemeinsam mit Jürgen Mittelstraß geschriebener Arbeiten zu Platon und Leibniz, Berlin/New York 2011.
} 
Da sind zum einen die von der Deutschen Vereinigung für Mathematische Logik und Grundlagenforschung der exakten Wissenschaften - damals unter dem Vorsitz von Hans Hermes (Münster/Westf.) und Arnold Schmidt (Marburg) - organisierten Kolloquien in Oberwolfach. Am ersten dieser Kolloquien (14.4. - 17.4. 1964), zu dem auch ich eingeladen war, trug Lorenzen im Kontext seiner Arbeit an der Monographie Differential und Integral (Frankfurt/M. 1965) zum Thema „Konstruktive Grundlagen der klassischen Analysis" vor. Zum anderen fand gleich anschließend (19. 4. 22. 4. 1964) ein von Lorenzen initiiertes und von ihm geleitetes Kolloquium über Philosophie, Logik und Mathematik in Gestalt einer von der Deutschen Forschungsgemeinschaft für ihre Stipendiaten und andere jüngere Wissenschaftler organisierten Veranstaltung im Waldhotel Glashütten/Taunus statt. Im Spätsommer schließlich reisten Lorenzen und ich gemeinsam zum International Congress for Logic, Methodology and Philosophy of Science nach Jerusalem (26. 8. - 2. 9. 1964). Zwischenlandung in Athen, dort Besuch der Akropolis, auf der Agora mit einem imaginierten Zeitsprung ins Athen Platons, und Lorenzen bereit zu einer Rede, Platon zu ehren - ein unvergeßlicher Moment. Der für die Kongreßplanung in Israel verantwortliche Kollege Yehoshua Bar-Hillel hatte Lorenzen eingeladen, die Leitung einer Sektion zu übernehmen, in der es um einen Disput gehen sollte: Axiomatisches versus konstruktives Vorgehen in den mathematisch-naturwissenschaftlichen Disziplinen. Als Kontrahenten hatte Lorenzen Abraham Robinson als Verteidiger axiomatischen Vorgehens und Friedrich Kambartel als Verteidiger konstruktiven Vorgehens gewinnen können. Die Teilnehmer wurden Zeugen eines modernen Paradigmas für vernünftige philosophische Auseinandersetzung. 9

In meiner Einleitung zur Festschrift anläßlich des 6o. Geburtstages von Paul Lorenzen am 24. März 1975 habe ich den damaligen Stand dieses Typs von Auseinandersetzungen auf der Seite der Verteidiger konstruktiven Vorgehens mit Worten zusammengefaßt, die ich noch immer für im wesentlichen zutreffend halte: „Die Zurückführung des theoretischen Geltungsproblems (die Frage nach dem `Sein $<$ und dem `Sollen`) auf technisches Können und praktisches Wollen ist für die Behandlung wissenschaftstheoretischer Fragestellungen in der konstruktiven Wissenschaftstheorie charakteristisch und verweist damit zugleich auf die große Nähe zum Pragmatismus in der ursprünglichen, an Kant und dem Common-Sensism orientierten Fassung bei C.S. Peirce. ${ }^{110}$

\footnotetext{
9 Vgl. Y. Bar-Hillel (ed.), Logic, Methodology and Philosophy of Science: Proceedings of the 1964 International Congress (Held at the Hebrew University of Jerusalem, Aug. 26-Sept. 2, under the Joint Auspices of the Division of Logic, Methodology and Philosophy of Science of the International Union of History and Philosophy of Science), Amsterdam 1965.

${ }^{10} \mathrm{~K}$. Lorenz (ed.), Konstruktionen versus Positionen: Beiträge zur Diskussion um die Konstruktive Wissenschaftstheorie I-II, Berlin/New York 1979, p. xIV.
} 
Open Access This chapter is licensed under the terms of the Creative Commons Attribution 4.0 International License (http://creativecommons.org/licenses/by/4.0/), which permits use, sharing, adaptation, distribution and reproduction in any medium or format, as long as you give appropriate credit to the original author(s) and the source, provide a link to the Creative Commons license and indicate if changes were made.

The images or other third party material in this chapter are included in the chapter's Creative Commons license, unless indicated otherwise in a credit line to the material. If material is not included in the chapter's Creative Commons license and your intended use is not permitted by statutory regulation or exceeds the permitted use, you will need to obtain permission directly from the copyright holder.

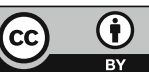

